\title{
Radiation pressure in massive star formation
}

\author{
Mark R. Krumholz ${ }^{1}$, Richard I. Klein ${ }^{2,3}$ \\ and Christopher F. McKee ${ }^{1,2}$ \\ ${ }^{1}$ Physics Department, University of California, Berkeley, Berkeley, CA 94720-7304 USA \\ emails: krumholz@astron.berkeley.edu,cmckee@astron.berkeley.edu \\ ${ }^{2}$ Astronomy Department, University of California, Berkeley, Berkeley, CA 94720-7304 USA \\ email: klein@astron.berkeley.edu \\ ${ }^{3}$ Lawrence Livermore National Laboratory, Livermore, CA 94550 USA
}

\begin{abstract}
Stars with masses of $\gtrsim 20 M_{\odot}$ have short Kelvin times that enable them to reach the main sequence while still accreting from their natal clouds. The resulting nuclear burning produces a huge luminosity and a correspondingly large radiation pressure force on dust grains in the accreting gas. This effect may limit the upper mass of stars that can form by accretion. Indeed, simulations and analytic calculations to date have been unable to resolve the mystery of how stars of $50 M_{\odot}$ and up form. We present two new ideas to solve the radiation pressure problem. First, we use three-dimensional radiation hydrodynamic adaptive mesh refinement simulations to study the collapse of massive cores. We find that in three dimensions a configuration in which radiation holds up an infalling envelope is Rayleigh-Taylor unstable, leading radiation driven bubbles to collapse and accretion to continue. We also present Monte Carlo radiative transfer calculations showing that the cavities created by protostellar winds provides a valve that allow radiation to escape the accreting envelope, further reducing the ability of radiation pressure to inhibit accretion.
\end{abstract}

Keywords. accretion, hydrodynamics, instabilities, radiative transfer, turbulence, methods: numerical, stars: formation

\section{Introduction}

Stars with masses $\gtrsim 20 M_{\odot}$ have short Kelvin times that enable them to reach the main sequence while still accreting (Shu et al. 1987). The resulting nuclear burning produces a huge luminosity and a correspondingly large radiation pressure force on dust grains in the incoming gas. This force can exceed the star's gravitational pull, possibly halting accretion and setting an upper limit on the star's final mass. Early spherically symmetric calculations found limits $\sim 20-40 M_{\odot}$ (Kahn 1974; Wolfire \& Cassinelli 1987) for typical galactic metallicities. More recent non-spherical calculations have loosened that constraint by considering the role of accretion disks (Nakano 1989; Nakano, Hasegawa, \& Norman 1995; Jijina \& Adams 1996), which concentrate the incoming matter into a smaller solid angle and also cast a shadow of reduced radiation pressure. Even with the effects of a disk, however, radiation pressure can still be a significant barrier to accretion. Numerical simulations by Yorke \& Sonnhalter (2002) found limiting masses of $\sim 40 M_{\odot}$ before radiation pressure reversed the inflow. However, observations show that considerably more massive stars exist, and their formation mechanism remains uncertain.

In this paper we present two new mechanisms that allow accretion onto massive stars to overcome the radiation pressure barrier and continue to higher masses. First, we perform $3 \mathrm{D}$ self-gravitational radiation hydrodynamic adaptive mesh refinement simulations of the collapse of massive protostellar cores, starting from both quiescent and turbulent initial conditions. We find that sufficiently massive stars create radiation-driven bubbles 
in accreting flows, but these bubbles become unstable and collapse due to a radiation Rayleigh-Taylor instability. We discuss the numerical methods we use in our simulations in $\S 2$, and we present the simulation results in $\S 3$. Our second new mechanism is protostellar winds, which we discuss in $\S 4$. Outflows launched from massive stars are essentially dust-free, and that as a result they are optically thin below their caps of sweptup material. We present Monte Carlo calculations of radiative transfer through envelopes with outflow cavities, and show that the cavities greatly reduce the radiation pressure on the accreting gas. These two new mechanisms indicate that radiation pressure is a much less significant barrier to massive star formation than previous work has suggested.

\section{Simulations}

\subsection{Numerical Methods}

In our simulations, we solve the equations of self-gravitational radiation hydrodynamics in the gray, flux-limited diffusion approximation. These are

$$
\begin{aligned}
\frac{\partial \rho}{\partial t}+\nabla \cdot(\rho \mathbf{v}) & =0 \\
\frac{\partial}{\partial t}(\rho \mathbf{v})+\nabla \cdot(\rho \mathbf{v} \mathbf{v}) & =-\nabla P-\rho \nabla \phi+\frac{\kappa_{\mathrm{R}, 0}}{c} \mathbf{F} \\
\frac{\partial}{\partial t}(\rho e)+\nabla \cdot[(\rho e+P) \mathbf{v}] & =-\rho \mathbf{v} \cdot \nabla \phi-\kappa_{\mathrm{P}, 0}(4 \pi B-c E)-\frac{\kappa_{\mathrm{R}, 0}}{c} \mathbf{v} \cdot \mathbf{F} \\
\nabla^{2} \phi & =4 \pi G \rho \\
\frac{\partial E}{\partial t}+\nabla \cdot \mathbf{F} & =\kappa_{\mathrm{P}, 0}(4 \pi B-c E)+\frac{\kappa_{\mathrm{R}, 0}}{c} \mathbf{v} \cdot \mathbf{F} \\
\mathbf{F}_{\mathbf{0}} & =-\frac{c \lambda\left(E_{0}\right)}{\kappa_{\mathrm{R}, 0}} \nabla E_{0},
\end{aligned}
$$

where $\rho, \mathbf{v}, P$, and $e$ are the density, velocity, pressure, and specific kinetic plus internal energy of the gas, $\phi$ is the gravitational potential, $\kappa_{\mathrm{R}}$ and $\kappa_{\mathrm{P}}$ are the Rosseland and Planck mean opacities, $E$ is the radiation energy density, $\mathbf{F}$ is the radiation flux, and $\lambda(E)$ is the flux limiter (Levermore \& Pomraning 1981). Subscript zeros indicate that quantities are evaluated in the frame co-moving with the gas.

The Berkeley adaptive mesh refinement code that we have developed (Truelove et al. 1998; Klein 1999; Howell \& Greenough 2003) consists of three modules. The hydrodynamics module updates the state with the hydrodynamic terms of the equations using a conservative Godunov scheme that is second-order accurate in both space and time. The gravity module then solves the Poisson equation for $\phi$ using a multigrid method. Finally, the radiation module performs a radiation update in two steps. It handles the radiation diffusion and emission/absorbtion terms $\left(\nabla \cdot \mathbf{F}\right.$ and $\left.\kappa_{\mathrm{P}}(4 \pi B-c E)\right)$ with an implicit method and the work/advection and force terms $\left(\kappa_{\mathrm{R}} \mathbf{F} / c\right.$ and $\left.\kappa_{\mathrm{R}} \mathbf{v} \cdot \mathbf{F} / c\right)$ with an explicit method. This approach has the advantage of minimizing the cost of the implicit solve, yet it does not cause instability because the diffusion and emission/abosbtion terms are larger than the work/advection terms by $O(c / v)$. The solvers all operate on adaptive grids (Berger \& Oliger 1984; Berger \& Collela 1989; Bell et al. 1994), allowing the calculations to reach an effective linear resolution up to 16,384 .

We represent any stars that form in the simulation using the Lagrangian sink particle algorithm of Krumholz, McKee, \& Klein (2004). The sink particles can move through the grid, accrete mass, and interact gravitationally with the gas. They also act as sources of radiation. We couple each sink particle to a protostellar model based on McKee \& 
Tan (2003), that updates the mass, radius, luminosity, and internal state of a star based on the accretion rate determined by the simulation. The model includes luminosity from accretion, Kelvin-Helmholtz contraction, deuterium burning, and hydrogen burning.

\subsection{Initial Conditions}

We simulate the formation of stars from both turbulent and quiescent massive pre-stellar cores. For quiescent runs we begin our simulations with spheres of gas of $100 M_{\odot}$ or $200 M_{\odot}$ with a density profile $\rho \propto r^{-2}$, a radius of $0.1 \mathrm{pc}$ or $0.2 \mathrm{pc}$, and temperature $40 \mathrm{~K}$, in solid body rotation with a ratio of rotational kinetic energy to gravitational potential energy $\beta=0.02$, the median observed for low mass cores (Goodman et al. 1993). The computational domain is a box $0.4 \mathrm{pc}$ or $0.6 \mathrm{pc}$ on a side for these runs, and the maximum resolution of these runs is $10 \mathrm{AU}$ and $14 \mathrm{AU}$, respectively. Note that these initial conditions are very similar to those used by Yorke \& Sonnhalter (2002).

For the turbulent initial conditions, we simulate a core based on the McKee \& Tan (2003) model. We use a density profile $\rho \propto r^{-2}$ continuing self-similarly to the edge of the computational domain, a box $0.8 \mathrm{pc}$ accross. The density profiel is normalized so that $150 M_{\odot}$ of gas lies within $0.1 \mathrm{pc}$ of the origin. We impose a Gaussian-random turbulent velocity field with its power on wavenumbers of $k=1-2$, where $k=1$ corresponds to a wavelength equal to the size of the computational domain. The Mach number $\mathcal{M}$ of the turbulence is chosen so that the kinetic and potential energies within the computational domain are equal, giving $\mathcal{M}=8.6$. The maximum resolution is $10 \mathrm{AU}$.

In our runs we refine any cell that is within 32 cells of a sink particle, where the gradient of radiation energy density satisfies $|\nabla E| / E>0.25$, or where the cell violates the Jeans condition for self-gravitational stability (Truelove et al. 1997).

\section{Simulation Results}

\subsection{Quiescent Initial Conditions}

The results of the $100 M_{\odot}$ and $200 M_{\odot}$ runs are qualitatively very similar, so for simplicity we only discuss them together. The accretion process appears to pass through three phases. During the first phase, when $M \lesssim 17 M_{\odot}$, the accretion flow is smooth and there are no obvious effects from radiation pressure. Low angular momentum gas accretes directly onto the star, and higher angular momentum gas falls into a Keplerian accretion disk. At $M \lesssim 5 M_{\odot}$ the accretion luminosity is dominant, but thereafter it becomes a relatively minor component of the total energy output.

The second phase begins at $\sim 17 M_{\odot}$, when the star is luminous enough for radiation pressure to start repelling gas. This leads to the formation of radiation-driven bubbles above and below the accretion disk, as shown in Figure 1, first panel. The bubbles are asymmetric above and below the accretion disk and with respect to the polar axis, an effect that earlier single-quadrant $2 \mathrm{D}$ simulations could not model. The asymmetry is the result of a positive feedback loop. If the radiation bubble has expanded farthest in a certain direction, then there is less gas above the star in that direction. As a result, that direction represents the path of minimum optical depth out of the core and radiation is collimated towards it. Since more radiation is flowing in that direction, there is a greater radiation pressure force, which in turn causes the bubble to expand even faster. At no point during the bubble phase does accretion stop, although it does become clumpy and erratic. Infalling gas that reaches a radiation bubble travels around its edge until it reaches the equatorial plane and the accretion disk. The disk itself is unaffected by the radiation pressure due to its high optical depth. This phase lasts $\sim 10^{4} \mathrm{yr}$. 


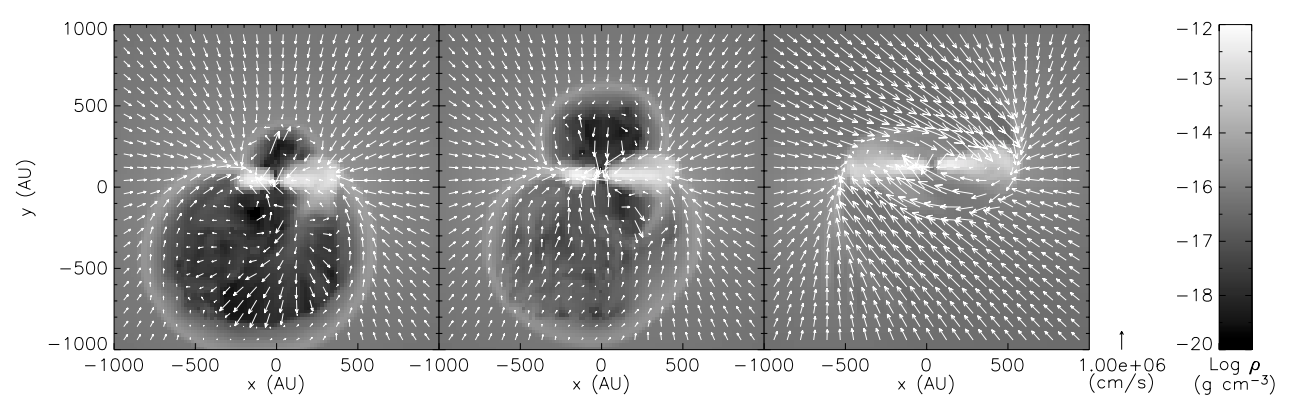

Figure 1. The plot shows a slice in the XZ plane at three different times, showing the initial growth, instability, and collapse of a radiation bubble. The times of the three slices are $1.5 \times 10^{4}$, $1.65 \times 10^{4}$, and $2.0 \times 10^{4} \mathrm{yrs}$, and the stellar masses are $21.3 M_{\odot}, 22.4 M_{\odot}$, and $25.7 M_{\odot}$.

The transition to the third phase of accretion happens at a mass of $22-26 M_{\odot}$, when the bubble becomes unstable and begins to collapse. The collapse of the bubble appears to occur when the bubble walls stop accelerating away from the star, so that there is a net gravitational force towards the star within the walls. The instability leads to ripples in the bubble walls, and eventually to gas breaking through into the bubble. This is shown in Figure 1, second panel. Physically, this process is easy to understand as RayleighTaylor instability: the gas is a heavy fluid, and radiation is in effect a light fluid. Once the local gravity is such that the radiation is below the gas, instability naturally results. However, the instability seems to begin with a large wavelength mode passing through the pole, in which the entire shell ripples. This mode cannot exist in an axisymmetric simulation, which is probably why previous work has not seen it.

Once enough gas has flooded in, the bubble effectively disappears, What is left is a remnant of the bubble wall. After this point, gas that falls towards the star is deflected to the side by the radiation field until it reaches the dense wall. The gas then flows along the wall into the accretion disk, and is able to accrete. This configuration is shown in Figure 1, third panel. To the point we have evolved the simulations thus far, this appears to be the final configuration of the system. However, it is possible that there may be more episodes of bubble formation and destruction. At the time of this writing, the simulations have reached a mass of $>27 M_{\odot}$, considerably larger than the $22.9 M_{\odot}$ Yorke \& Sonnhalter (2002) found for their calculations with gray radiative transfer. Our simulations are continuing to determine an upper mass limit.

\subsection{Turbulent Initial Conditions}

Our turbulent run shows results qualitatively quite similar to our queiscent runs. In the early stages of the simulation, a star with a disk around it forms. The orientation of the disk varies in time, as the angular momentum of the accreting gas changes in time due to the turbulence. At $17-18 M_{\odot}$, a bubble grows around the star. We have evolved the star to a mass of $\sim 25 M_{\odot}$ as of this writing. The star is still in the bubble phase, and the bubble shows no signs of collapsing yet, as it showed by this mass in the quiescent cases. The bubbles above and below the accretion disk have also grown to a diameters of almost $2000 \mathrm{AU}$, larger than they became in the quiescent case. We believe the changes in bubble evolution are probably due to a combination of the change in the normalization of the density profile $\left(150 M_{\odot}\right.$ versus $100 M_{\odot}$ within $\left.0.1 \mathrm{pc}\right)$ and to the turbulence, both of which reduce the effective radial infall velocity at the start of the bubble phase and thus allow the expansion of the bubble to accelerate for longer times. The mean accretion rate in the simulation thus far is $2.8 \times 10^{-3} M_{\odot} \mathrm{yr}^{-1}$. Because our initial conditions are 
not in virial balance on small scales, this is slightly larger than the $1.6 \times 10^{-3} M_{\odot} \mathrm{yr}^{-1}$ that McKee \& Tan (2003) predict for our initial density profile.

We do not see any evidence of fragmentation to form many stars, as found by Dobbs, Bonnell, \& Clark (2005) in simulations with fairly similar initial conditions. We hypothesize that the different outcome stems from our more detailed treatment of the radiation. Dobbs et al. use either an isothermal or a barotropic equation of state, and find that the barotropic equation of state reduces their number of fragments from tens to a few. This points to the importance of heating in determining whether fragmentation occurs. However, even a barotropic equation of state misses the vast majority of the heating. Since neither our simulations nor those of Dobbs et al. resolve the protostellar radius, most of the energy released by the infalling gas is released at sub-grid scales, in the final plunge onto the stellar surface. Our method captures this energy, by including the accretion luminosity in the energy output by our sink particle, but a barotropic equation of state omits it. In our simulations, the high accretion luminosity that results from the high pressure in the protostellar core heats the gas within $\sim 1000$ AU of the star (where Dobbs et al. get fragmentation) to hundreds of $\mathrm{K}$ in only a thousand years. This is shorter than the free-fall time of $\sim 4000 \mathrm{yr}$ at $1000 \mathrm{AU}$, so the heating prevents fragmentation.

\section{Protostellar Outflows}

We also consider the effects of protostellar outflows on the accretion process (Krumholz, McKee, \& Klein 2005 for a detailed discussion). Beuther, Schilke, \& Geuth (2004) report interferometric measurements showing outflows from massive protostars with collimation factors of $\sim 2$ up to $\sim 10$. They conclude that high and low mass outflows have similar collimation factors, typically $2-5$. (The collimation factor is the ratio of the outflow's length to its width.) Richer et al. (2000) show that the momentum of CO outflows driven by massive stars scales with the bolometric luminosity of the source in the same manner as for low mass stars. From these observations, the natural conclusion is that low and high mass stellar outflows have a common driving mechanism and similar morphologies.

This analysis suggests that massive protostellar outflows originate near their source stars, inside the stars' dust destruction radius. As a result, they should be dust-free when launched. Once the outflowing gas is far enough from the star, grains can begin to grow. However, the wind density is relatively low and its speed is very high. For a range of possible shapes of the cavity filled by outflowing gas, Krumholz et al. show that the largest grains ejected in the wind from a $50 M_{\odot}$ star at a typical velocity of $1000 \mathrm{~km}$ $\mathrm{s}^{-1}$ reach sizes of no more than $\sim 10^{-4} \mu \mathrm{m}$ before escaping the accreting core. Since the optical depth of grains to radiation both from the star and the envelope obeys $\kappa \propto r_{\text {gr }}$, this means that the outflow channel will be quite optically thin up to its cap of swept-up material. Since the surrounding envelope is very optically thick, we expect the outflow cavity to collimate the radiation and carry it away from the accreting gas.

To see how this affects radiation pressure, we compute radiative transfer through envelopes witih and without outflow cavities using the Monte Carlo code of Whitney et al. $(2003 \mathrm{a}, \mathrm{b})$, and then compute radiation pressure forces within the envelope using a ray tracing code. We find that even in a very low surface density envelope with $\Sigma \approx 0.1 \mathrm{~g}$ $\mathrm{cm}^{-3}$, an outflow cavity reduces the radiation pressure force in the equatorial plane by a factor of $\sim 5$. At the higher surface densities typical of most massive star forming regions, the collimation is even more effective, and the radiation pressure is reduced by a factor of $\gtrsim 10$. Since observations show that such outflows are ubiquitous, we expect suggest that radiation pressure is a much less significant barrier to massive star formation than calculations without protostellar outflows have found. 


\section{Conclusions}

Massive star formation by accretion is much easier than previous work suggested. In three dimensions, radiation holding up accreting gas is subject to Rayleigh-Taylor instability. Radiation deflects gas into optically thick fingers that accrete onto the star, while the radiation diffuses out around the compressed gas. Differing initial conditions, including ones with turbulence, do not appear to change this basic evolution. Furthermore, even without this instability, the outflows from massive stars are by themselves very effective at collimating radiation and carrying it away from the accreting gas. Outflows cavities produce order-of-magnitude reductions in the radiation pressure force experienced by inflowing gas, and thus make accretion even easier. We therefore conclude that radiation pressure cannot prevent the formation of massive stars by accretion.

\section{Acknowledgements}

This work received financial and computing support from: NASA GSRP grant NGT 2-52278 (MRK); the US Department of Energy at the Lawrence Livermore National Laboratory under the auspices of contract W-7405-Eng-48 (RIK and MRK); NASA ATP grant NAG 5-12042 (RIK and CFM); NSF grant AST-0098365 (CFM); the NSF San Diego Supercomputer Center through NPACI program grant UCB267 (all); and the National Energy Research Scientific Computing Center, which is supported by the Office of Science of the U.S. Department of Energy under Contract No. DE-AC03-76SF00098, through ERCAP grant 80325 (all).

\section{References}

Bell, J., Berger, M., Saltzman, J., \& Welcome, M. 1994, SIAM J. Sc. Comp., 15, 127

Berger, M. J., \& Collela, P. 1989, J. Comp. Phys., 82, 64

Berger, M. J., \& Oliger, J. 1984, J. Comp. Phys., 53, 484

Beuther, H., Schilke, P., \& Gueth, F. 2004, ApJ, 608, 330

Dobbs, C. L., Bonnell, I. A., \& Clark, P. C. 2005, MNRAS, in press, astro-ph/0502479

Goodman, A. A., Benson, P. J., Fuller, G. A., \& Myers, P. C. 1993, ApJ, 406, 528

Howell, L. H., \& Greenough, J. A. 2003, J. Comp. Phys., 184, 53

Jijina, J., \& Adams, F. C. 1996, ApJ, 462, 874

Kahn, F. D. 1974, A\&A, 37, 149

Klein, R. I. 1999, J. Comp. App. Math., 109, 123

Krumholz, M. R., McKee, C. F., \& Klein, R. I. 2004, ApJ, 611, 399

Krumholz, M. R., McKee, C. F., \& Klein, R. I. 2005, ApJ, 618, L33

Levermore, C. D., \& Pomraning, G. C. 1981, ApJ, 248, 321

McKee, C. F., \& Tan, J. C. 2003, ApJ, 585, 850

Nakano, T. 1989, ApJ, 345, 464

Nakano, T., Hasegawa, T., \& Norman, C. 1995, ApJ, 450, 183

Richer, J.S., Shepherd, D.S., Cabrit, S., Bachiller, R., \& Churchwell, E. 2000, in "Protostars and Planets IV", eds. V. Mannings, A.P. Boss, S.S. Russell, (Tucson: The University of Arizona Press), p. 867

Shu, F. H., Adams, F. C., \& Lizano, S. 1987, ARAE A, 25, 23

Truelove, J. K., Klein, R. I., McKee, C. F., Holliman, J. H., II, Howell, J. H., \& Greenough, J. A. $1997, A p J, 489, \mathrm{~L} 179$

Truelove, J. K., Klein, R. I., McKee, C. F., Holliman, J. H., Howell, L. H., Greenough, J. A., \& Woods, D. T. 1998, ApJ, 495, 821

Whitney, B. A., Wood, K., Bjorkman, J. E., \& Cohen, M. 2003a, ApJ, 598, 1079

Whitney, B. A., Wood, K., Bjorkman, J. E., \& Wolff, M. J. 2003b, ApJ, 591, 1049

Wolfire, M. G., \& Cassinnelli, J. P. 1987, ApJ, 319, 850

Yorke, H. W., \& Sonnhalter, C. 2002, ApJ, 659, 846 